\title{
A Note On Cheap Talk and Burned Money*
}

\author{
Navin Kartik ${ }^{\dagger}$ \\ Department of Economics \\ University of California, San Diego
}

First version: March 2005

This version: July 2006

\begin{abstract}
Austen-Smith and Banks (Journal of Economic Theory, 2000) study how money burning can expand the set of pure cheap talk equilibria of Crawford and Sobel (Econometrica, 1982). I identify an error in the main Theorem of Austen-Smith and Banks, and provide a variant that preserves some of the important implications. I also prove that cheap talk can be influential with money burning if and only if it can be influential without money burning. This strengthens a result of Austen-Smith and Banks, but uncovers other errors in their analysis. Finally, an open conjecture of theirs is proved correct.
\end{abstract}

Keywords: Cheap Talk, Money Burning, Signaling

J.E.L. Classification: C7, D8

Running Title: On Cheap Talk and Burned Money

*I thank Vince Crawford, Joel Sobel, and two anonymous referees for helpful comments.

${ }^{\dagger}$ Email: nkartik@ucsd.edu; Web: http://econ.ucsd.edu/ nkartik; Address: 9500 Gilman Drive, La Jolla, CA 92093-0508. 


\section{Introduction}

In an important paper on signaling with multiple instruments, Austen-Smith and Banks [2, hereafter ASB] augment the seminal cheap talk model of Crawford and Sobel [3, hereafter CS] by allowing the Sender to send not only costless messages, but also choose from a set of purely dissipative signals, i.e. "burn money". ASB's contribution is twofold: first, to show that money burning by itself can be an effective signaling instrument in the CS setting; second, to study how money burning can interact with and influence the informativeness of cheap talk messages.

This note accomplishes three tasks:

1. Section 3 identifies an error in Theorem 1 of ASB that asserts the existence of particular equilibria with money burning in relation to equilibria of CS. I provide a variant of the Theorem, which preserves some of the main implications, but not all of them.

2. Section 4 derives a result showing that money burning cannot expand the set of environments in which cheap talk is credible, except perhaps in knife-edged cases. This considerably strengthens Theorem 2 in ASB, but also shows that ASB's (p. 13) claims following that Theorem are incorrect. In particular, not only is the example reported in ASB (p. 13) erroneous, ${ }^{1}$ but moreover, the result here implies that no such generic example exists.

3. Suppose the maximal amount of available burned money is some $\bar{b} \geq 0$. If $\bar{b}=0$, the setting is effectively that of CS. Throughout their paper, ASB work with the case of $\bar{b}=\infty$ (or sufficiently large). However, ASB (p. 15) conclude with a conjecture that the "qualitative properties of the equilibrium set are close to those of the CS model" when $\bar{b} \approx 0$; to my knowledge, this has remained an open question. In Section 5 , I show that the conjecture is in fact correct and thereby establish a continuity result on the equilibrium correspondence at $\bar{b}=0$.

\footnotetext{
${ }^{1}$ Footnote 8 in this note points out exactly where their example fails.
} 
Before turning to the formalities, I should mention that the ASB model can be considered as a limit of the discriminatory signaling model studied in Kartik [4]. ${ }^{2}$ An earlier, extended version of this note [5] contains a detailed comparison of the similarities and differences, and also discusses issues pertaining to refinement of equilibria in the ASB model.

\section{Preliminaries}

To preserve continuity of exposition, I follow ASB's notation closely; the reader should consult their paper for a discussion of the model.

A Sender, $S$, is privately informed about a variable, $t \in[0,1]$ (his type) which is drawn from a distribution with density $h, h(t)>0$ for all $t$. $S$ sends a signal to the Receiver, $R$, who observes the signal and then takes an action $a \in \mathbb{R}$. Let $\sigma:[0,1] \rightarrow M \times \mathbb{R}_{+}$be the Sender's (pure) strategy that consists of a cheap talk message $m \in M$, where $M$ is any uncountable space, and a burned money component $b \in \mathbb{R}_{+}$, for every type $t \in[0,1]$. Let $\alpha: M \times \mathbb{R}_{+} \rightarrow \mathbb{R}_{+}$be the Receiver's (pure) strategy that consists of an action $a \in \mathbb{R}$ for every $(m, b)$ pair. The Receiver's beliefs are denoted by the $\operatorname{cdf} G(\cdot \mid m, b)$. Over triplets $(a, b, t)$, the Receiver's preferences are $u^{R}(a, t)$ and the Sender's preferences are $u^{S}(a, t)-b$ where $u^{S}$ and $u^{R}$ satisfy the CS assumptions. ${ }^{3}$ The utility maximizing actions given $t$ are denoted $y^{i}(t) \equiv \arg \max _{a} u^{i}(a, t)$ for each $i \in\{S, R\}$; it is assumed that for all $t, y^{R}(t)<y^{S}(t)$. For any $t \leq t^{\prime}$, define

$$
y\left(t, t^{\prime}\right) \equiv \begin{cases}\arg \max _{a} \int_{t}^{t^{\prime}} u^{R}(a, \tau) h(\tau) d \tau & \text { if } t^{\prime}>t \\ y^{R}(t) & \text { if } t^{\prime}=t\end{cases}
$$

As shorthand, let $y(t) \equiv y(t, t)$.

In what follows, I use two concepts from CS. First, recall the idea of a solution to the standard arbitrage condition.

\footnotetext{
${ }^{2}$ By definition, money burning is non-discriminatory in the sense that its cost does not vary with the Sender's private information or type; this is in contrast to discriminatory signaling where the cost of using a particular signal varies with the Sender's type.

${ }^{3}$ That is, for each $i \in\{S, R\}, u^{i}(\cdot, \cdot)$ is twice-differentiable, $u_{11}^{i}(\cdot, \cdot)<0$, and $u_{12}^{i}(\cdot, \cdot)>0$. To ease notation, I have suppressed the bias parameter, $x$, used by ASB.
} 
Definition 1. A sequence $\left\langle s_{0}, s_{1}, \ldots, s_{N}\right\rangle$ such that

$$
\forall i=1, \ldots, N-1, \quad u^{S}\left(y\left(s_{i-1}, s_{i}\right), s_{i}\right)=u^{S}\left(y\left(s_{i}, s_{i+1}\right), s_{i}\right)
$$

is a forward (resp. backward) solution to (A) if $s_{1}>s_{0}$ (resp. $\left.s_{1}<s_{0}\right)$.

Next, CS (p. 1444) introduced a condition on the product space of preferences and distribution of private information that ensures the difference equation solutions to the above arbitrage condition satisfy a "regularity" property.

Condtion M. For any two increasing sequences, $\left\langle t_{0}, t_{1}, \ldots, t_{K}\right\rangle$ and $\left\langle\tilde{t}_{0}, \tilde{t}_{1}, \ldots, \tilde{t}_{K}\right\rangle$, that are both forward solutions to (A), if $t_{1}>\tilde{t}_{1}>t_{0}=\tilde{t}_{0}$, then $t_{j}>\tilde{t}_{j}$ for all $j \in\{1, \ldots, K\}$.

What this says is that if we start at a given point, the solutions to (A) must all move up or down together.

Throughout, the term equilibrium refers to a sequential equilibrium, which is equivalent to perfect Bayesian equilibrium in signaling games such as this one.

\section{Squeezing in Separating Segments}

ASB (Theorem 1, p. 7) assert the following.

ASB Theorem. Let $(\sigma, \alpha)$ be a CS equilibrium with supporting partition $\left\langle t_{0} \equiv 0, t_{1}, \ldots, t_{N} \equiv 1\right\rangle$.

Then for all $\hat{t} \leq t_{1}$, there exists a partition $\left\langle s_{0} \equiv 0, s_{1} \equiv \hat{t}, \ldots, s_{N}, s_{N+1} \equiv 1\right\rangle$ supporting an equilibrium $(\sigma, \alpha)(\hat{t})$ such that

$$
\begin{aligned}
& \forall i=0 \ldots, N-1, \quad \forall t \in\left[s_{i}, s_{i+1}\right), \quad \sigma(t)=\left(m_{i}, 0\right), \quad m_{i} \neq m_{j} \quad \forall i \neq j ; \\
& \forall t \in\left[s_{N}, 1\right], \quad \sigma(t)=\left(m^{\circ}, b(t)\right),
\end{aligned}
$$

where $b(t)$ is a strictly increasing function. ${ }^{4}$

\footnotetext{
${ }^{4} \mathrm{ASB}$ also pin down the function $b(t)$, which I do not include here for brevity.
} 


\subsection{The Problem}

ASB's proof proceeds in two steps. In the first, they start by picking any $\hat{t}<t_{1}$ (the case of $\hat{t}=t_{1}$ can be dealt with easily), and consider a forward solution to (A) starting with $s_{0} \equiv 0$ and $s_{1} \equiv \hat{t}$. This, they claim, provides a sequence $\left\langle s_{0} \equiv 0, s_{1} \equiv \hat{t}, \ldots, s_{N}\right\rangle$ such that $s_{n}<t_{n}$ for all $n \in\{1, \ldots, N\}$. Their justification of this claim contains the error. The second part of the proof is to construct the strictly increasing function $b(t)$ such that it is optimal for all types $t \in\left[s_{N}, 1\right]$ to reveal themselves by burning $b(t)$.

I note that if the above claim were true, that would make Condition $M$ always true, since the monotonicity of forward solutions to (A) is precisely what it assumes. The specific error leading to ASB's assertion is the following. On p. 8, they define for any $s^{\prime}, s$, and $t$, the function

$$
V\left(s^{\prime}, s, t\right)=u^{S}\left(y\left(s^{\prime}, s\right), s\right)-u^{S}(y(s, t), s)
$$

ASB claim that fixing $s^{\prime}$ and setting $V \equiv 0$ yields their Eq. (6) through implicit differentiation:

$$
\left.\frac{d t}{d s}\right|_{s^{\prime}}=\frac{u_{2}^{S}\left(y\left(s^{\prime}, s\right), s\right)-u_{2}^{S}(y(s, t), s)}{u_{1}^{S}(y(s, t), s) y_{2}(s, t)}
$$

But this is wrong: it ignores the indirect effect of $s$ on $V$ through the change of $y\left(s^{\prime}, s\right)$ and $y(s, t)$. To see this, observe that totally differentiating $V$ with respect to $s$ and $t$ (holding $s^{\prime}$ fixed) yields

$$
\begin{aligned}
d V= & {\left[u_{1}^{S}\left(y\left(s^{\prime}, s\right), s\right) y_{2}\left(s^{\prime}, s\right)+u_{2}^{S}\left(y\left(s^{\prime}, s\right), s\right)-u_{1}^{S}(y(s, t), s) y_{1}(s, t)-u_{2}^{S}(y(s, t), s)\right] d s } \\
& -\left[u_{1}^{S}(y(s, t), s) y_{2}(s, t)\right] d t
\end{aligned}
$$

and therefore the correct formula is

$$
\left.\frac{d t}{d s}\right|_{s^{\prime}}=\frac{u_{2}^{S}\left(y\left(s^{\prime}, s\right), s\right)-u_{2}^{S}(y(s, t), s)}{u_{1}^{S}(y(s, t), s) y_{2}(s, t)}+\frac{u_{1}^{S}\left(y\left(s^{\prime}, s\right), s\right) y_{2}\left(s^{\prime}, s\right)-u_{1}^{S}(y(s, t), s) y_{1}(s, t)}{u_{1}^{S}(y(s, t), s) y_{2}(s, t)}
$$

For ASB's claim to go through, it would have to be that the Right Hand Side (RHS) of Eq. $\left(6^{*}\right)$ is positive. As they argue, the first term indeed is: both numerator and denominator of the fraction are negative. However, the second term in $\left(6^{*}\right)$ - which is missing in (6) - is negative. To see this, first note that the denominator is negative, just as in the first term. In 
the numerator, $y_{1}(\cdot, \cdot)>0$ and $y_{2}(\cdot, \cdot)>0$, but $u_{1}^{S}\left(y\left(s^{\prime}, s\right), s\right)>0$ whereas $u_{1}^{S}(y(s, t), s)<0$. Hence the numerator is positive, whereby the whole second term is negative. Accordingly, one cannot in general sign the RHS of $\left(6^{*}\right)$, leading to a failure of the argument of ASB.

There is at least one explicit conclusion that ASB draw from their Theorem that may not be correct. ASB (p. 11) say that their result "implies that a sufficient condition for there to exist equilibria exhibiting both influential cheap talk and influential costly signals is that there exist influential CS equilibria." Given the error, it is an open question whether this is true when Condition M does not hold. A corollary to Theorem 1 below is that a sufficient condition is that there exists a CS equilibrium with three influential messages. ${ }^{5}$

\subsection{A Correct Variant}

ASB's Theorem 1 is valid under Condition M. ${ }^{6}$ However, one would like to know whatif anything - can be said without imposing Condition M, for at least two reasons: first, the results of CS regarding existence and characterization of pure cheap talk equilibria do not require Condition $\mathrm{M}$; second, Condition $\mathrm{M}$ is not a condition on primitives. ${ }^{7}$ There are a few ways one might alter ASB's Theorem in this vein; I provide one below which arguably preserves their main points. As I understand it, ASB's primary goal was to show that "we can squeeze in separating segments at the far end of any CS partition." (p. 7, their emphasis) Their Theorem however claimed more: not only can we squeeze in a separating segment at the far end of a CS partition, but moreover, we can squeeze it in while maintaining the same number of influential cheap talk messages. It is here that one runs into difficulty. Instead, if we are satisfied with squeezing in separation at the cost of reducing the number of influential cheap talk messages by one, this can be done. Formally,

Theorem 1. Let there be a CS equilibrium with supporting partition $\left\langle t_{0} \equiv 0, t_{1}, \ldots, t_{N} \equiv 1\right\rangle$.

\footnotetext{
${ }^{5}$ A precise definition of influential messages is postponed to Section 4.

${ }^{6}$ In most applications of CS, Condition M is typically satisfied. For example, it holds in the widely-used "uniform quadratic" setting where the prior is uniform and utilities are quadratic loss functions.

${ }^{7}$ See Theorem 2 in CS for sufficient conditions on primitives that imply Condition M.
} 
Then there exists an equilibrium $(\sigma, \alpha)$ such that

$$
\begin{aligned}
& \forall i=0 \ldots, N-2, \quad \forall t \in\left[t_{i}, t_{i+1}\right), \quad \sigma(t)=\left(m_{i}, 0\right), \quad m_{i} \neq m_{j} \quad \forall i \neq j ; \\
& \forall t \in\left[t_{N-1}, 1\right], \quad \sigma(t)=\left(m^{\circ}, b(t)\right),
\end{aligned}
$$

where $b(t)$ is a strictly increasing function.

The proof is relegated to the Appendix since the logic is similar to that of ASB's Theorem 1. This modification of the ASB Theorem preserves the essence of their result. In particular, it immediately implies that full revelation is an equilibrium outcome.

Corollary 1. There is an equilibrium $(\sigma, \alpha)$ such that for all $t, \alpha(\sigma(t))=y(t)$.

Proof. Apply Theorem 1 to a CS "babbling" equilibrium, i.e. a CS equilibrium with supporting partition $\left\langle t_{0} \equiv 0, t_{1} \equiv 1\right\rangle$.

It should be noted that this Corollary is weaker than ASB's (p. 11) Corollary 1, which is correct despite the error in their Theorem 1.

\section{Can Money Burning Make Cheap Talk Influential?}

Following ASB, say that an equilibrium has influential cheap talk if a particular level of money burning can elicit multiple actions in equilibrium through distinct accompanying cheap talk messages.

Definition 2. An equilibrium $(\sigma, \alpha)$ has influential cheap talk if there exist $t$ and $t^{\prime}$ such that $m(t) \neq m\left(t^{\prime}\right), b(t)=b\left(t^{\prime}\right)$, and $\alpha(\sigma(t)) \neq \alpha\left(\sigma\left(t^{\prime}\right)\right)$.

Similarly, a CS equilibrium is said to be influential if at least two different Receiver actions are played on the equilibrium path.

Theorem 2 of ASB shows that certain kind of equilibria with influential cheap talk (termed "left-pooling influential equilibria") exist if and only if influential equilibria exist in CS. However, following their Theorem 2, ASB (p. 13) claim that their Theorem "cannot 
be extended to cover all influential equilibria" (their emphasis), because there may be some equilibria with influential cheap talk when there are no influential CS equilibria. To support this claim, ASB (p. 13) construct an example where there assert that there is no influential CS equilibrium, but they claim an equilibrium with influential cheap talk in the presence of burned money. Unfortunately, the example is erroneous: the strategy profile they report is not an equilibrium. ${ }^{8}$ The following result implies that generically, one cannot construct such an example, because except in knife-edged cases, an equilibrium with influential cheap talk exists in the ASB model if and only if an influential equilibrium exists in CS. ${ }^{9}$

Theorem 2. Assume $u^{S}(y(0), 0) \neq u^{S}(y(0,1), 0)$. There exists an equilibrium with influential cheap talk if and only if there exists an influential CS equilibrium.

That is, contrary to their assertion, ASB's Theorem 2 does extend to all influential equilibria, except perhaps in non-generic cases where type 0 is exactly indifferent between actions $y(0)$ and $y(0,1)$. The proof of the Theorem requires two Lemmas, the latter of which holds independent interest outside the ASB model.

Lemma 1. If there is an equilibrium with influential cheap talk, then there exists a strictly increasing sequence, $\left\langle t_{1}, t_{2}, t_{3}\right\rangle$, that satisfies $(A)$.

Proof. Suppose that $(\sigma, \alpha)$ is an equilibrium with influential cheap talk. Then there exist $t^{\prime}$, $t^{\prime \prime}, m^{\prime}, m^{\prime \prime}$, and $b$ such that $\sigma\left(t^{\prime}\right)=\left(m^{\prime}, b\right), \sigma\left(t^{\prime \prime}\right)=\left(m^{\prime \prime}, b\right)$, and $\alpha(\sigma(t \prime))<\alpha\left(\sigma\left(t^{\prime \prime}\right)\right)$. Let $a_{i} \equiv \alpha\left(\sigma\left(t^{\prime}\right)\right), a_{k} \equiv \alpha\left(\sigma\left(t^{\prime \prime}\right)\right)$, and $a_{j} \equiv \inf _{a>a_{i}}\left\{a: a \in \bigcup_{t \in[0,1]} \alpha(\sigma(t))\right\} . \quad$ For $l=i, j, k$, let $t_{l} \equiv \inf \left\{t: \alpha(\sigma(t))=a_{l}\right\}$ and $t_{l+1} \equiv \sup \left\{t: \alpha(\sigma(t))=a_{l}\right\}$. By Lemma 1 of ASB, $t_{i}<t_{i+1} \leq t_{j} \leq t_{j+1} \leq t_{k}<t_{k+1}$, and by construction $t^{\prime} \in\left[t_{i}, t_{i+1}\right]$ and $t^{\prime \prime} \in\left[t_{k}, t_{k+1}\right]$. If $t_{i+1}=t_{k}$, then we are done, since $\left\langle t_{i}, t_{i+1}, t_{k+1}\right\rangle$ satisfies $(\mathrm{A})$.

So suppose $t_{i+1}<t_{k}$. If $b(\cdot)$ is non-decreasing on $\left(t_{i}, t_{k+1}\right)$, then $b(t)=b$ for all $t \in\left(t_{i}, t_{k+1}\right)$, in which case $t_{j}=t_{i+1}$, and we are done because $\left\langle t_{i}, t_{i+1}, t_{j+1}\right\rangle$ satisfies (A).

\footnotetext{
${ }^{8}$ The reason it is not an equilibrium is that all types in $[0.15,0.2)$ would strictly prefer to deviate from the prescribed strategy and play $\sigma(0.2)$ instead. This is because type 0.15 's ideal action is $0.15+0.1157=0.2657$ which is closer to $\alpha(\sigma(0.2))=0.2889$ than $\alpha(\sigma(0.15))=0.1739$.

${ }^{9} \mathrm{~A}$ working paper version of ASB [1] contained this result for the "uniform quadratic" special case.
} 
It remains to consider $t_{i+1}<t_{k}$ and $b(\cdot)$ decreasing somewhere on $\left(t_{i}, t_{k+1}\right)$. By Lemma 1 of $\mathrm{ASB}$, there exists some $t_{n} \in\left(t_{i}, t_{k+1}\right)$ such that $b(\cdot)$ is discontinuous at $t_{n}$, and $b^{n} \equiv \lim _{\varepsilon \downarrow 0} b\left(t_{n}-\varepsilon\right)>b_{n} \equiv \lim _{\varepsilon \downarrow 0} b\left(t_{n}+\varepsilon\right)$. It is straightforward that there must be some $t>t_{n}$ such that $b(\cdot)$ is pooling on $\left(t_{n}, t\right)$ - if not, for small enough $\varepsilon>0$, a type $t_{n}-\varepsilon$ has a profitable deviation to $\sigma\left(t_{n}+\varepsilon\right)$. Let $a_{n} \equiv \min _{a>y\left(t_{n}\right)}\left\{a: a \in \bigcup_{t \in[0,1]} \alpha(\sigma(t))\right\}$, $t_{n+1} \equiv \sup \left\{t: \alpha(\sigma(t))=a_{n}\right\}$, and $\left(m_{n}, b_{n}\right) \equiv \sigma(t)$ for any $t \in\left(t_{n}, t_{n+1}\right)$. There are two cases: either (i) for some $\delta>0, b(\cdot)$ is separating on $\left(t_{n}-\delta, t_{n}\right)$; or (ii) for some $\delta>0, b(\cdot)$ is pooling on $\left(t_{n}-\delta, t_{n}\right)$.

(i) It is straightforward to verify that the following incentive compatibility condition must hold:

$$
u^{S}\left(y\left(t_{n}, t_{n+1}\right), t_{n}\right)-b_{n}=u^{S}\left(y\left(t_{n}\right), t_{n}\right)-b^{n}
$$

By continuity, it follows that for some $t_{n-1}<t_{n}$, and $\varepsilon>0$ such that $b^{n}>b_{n}+\varepsilon$,

$$
u^{S}\left(y\left(t_{n}, t_{n+1}\right), t_{n}\right)-\left(b_{n}+\varepsilon\right)=u^{S}\left(y\left(t_{n-1}, t_{n}\right), t_{n}\right)-b^{n}
$$

Since $b^{n}>b_{n}+\varepsilon$, we have $u^{S}\left(y\left(t_{n-1}, t_{n}\right), t_{n}\right)>u^{S}\left(y\left(t_{n}, t_{n+1}\right), t_{n}\right)$, and consequently, $y\left(t_{n}, t_{n+1}\right)>y^{S}\left(t_{n}\right)>y\left(t_{n-1}, t_{n}\right)$. By continuity, there exists some $\tilde{t} \in\left(t_{n}, t_{n+1}\right)$ such that $u^{S}\left(y\left(t_{n-1}, t_{n}\right), t_{n}\right)=u^{S}\left(y\left(t_{n}, \tilde{t}\right), t_{n}\right)$, and consequently, $\left\langle t_{n}, \tilde{t}, t_{n+1}\right\rangle$ satisfies (A).

(ii) Let $a_{n-1} \equiv \max _{a<y\left(t_{n}\right)}\left\{a: a \in \bigcup_{t \in[0,1]} \alpha(\sigma(t))\right\}$, and $t_{n-1} \equiv \inf \left\{t: \alpha(\sigma(t))=a_{n-1}\right\}$. Then, the following incentive compatibility condition must hold:

$$
u^{S}\left(y\left(t_{n}, t_{n+1}\right), t_{n}\right)-b_{n}=u^{S}\left(y\left(t_{n-1}, t_{n}\right), t_{n}\right)-b^{n}
$$

Since $b^{n}>b_{n}$, we have $u^{S}\left(y\left(t_{n-1}, t_{n}\right), t_{n}\right)>u^{S}\left(y\left(t_{n}, t_{n+1}\right), t_{n}\right)$, and consequently, $y\left(t_{n}, t_{n+1}\right)>y^{S}\left(t_{n}\right)>y\left(t_{n-1}, t_{n}\right)$. By continuity, there exists some $\tilde{t} \in\left(t_{n}, t_{n+1}\right)$ such that $u^{S}\left(y\left(t_{n-1}, t_{n}\right), t_{n}\right)=u^{S}\left(y\left(t_{n}, \tilde{t}\right), t_{n}\right)$, and consequently, $\left\langle t_{n}, \tilde{t}, t_{n+1}\right\rangle$ satisfies $(\mathrm{A})$.

The next Lemma says that generically, a sufficient condition for the existence of an influential CS equilibrium is that there exist some non-trivial forward solution to (A). Crucially, this forward solution need not start at the lower end of the type space, 0 , nor end at the upper endpoint, 1. This result may be useful in applications of CS more generally, 
because it provides a sufficient condition for influential CS equilibria when Condition M does not hold. ${ }^{10}$

Lemma 2. Assume $u^{S}(y(0), 0) \neq u^{S}(y(0,1), 0)$. If there exists a strictly increasing sequence, $\left\langle t_{i-1}, t_{i}, t_{i+1}\right\rangle$, that satisfies $(A)$, then there exists an influential CS equilibrium.

Proof. I start by arguing that there is a forward solution to $(\mathrm{A}),\left\langle t_{i-1}, \hat{t}, 1\right\rangle$, such that $\hat{t} \in\left[t_{i}, 1\right)$. Clearly this is true if $t_{i+1}=1$, so assume that $t_{i+1}<1$. For any $\tau_{1}>t_{i-1}$, there is at most one value of $\tau_{2}>\tau_{1}$ such that the sequence $\left\langle t_{i-1}, \tau_{1}, \tau_{2}\right\rangle$ satisfies (A), due to the monotonicity of $y(\cdot, \cdot)$ in each argument and the concavity of $u^{S}(\cdot, \cdot)$ in its first argument. Moreover, since $u^{S}(y(t, 1), 1)<u^{S}(y(1), 1)$ for all $t<1$, continuity implies that there exists some $\tau_{1} \in\left(t_{i}, 1\right)$ for which there is no $\tau_{2}>\tau_{1}$ such that $\left\langle t_{i-1}, \tau_{1}, \tau_{2}\right\rangle$ satisfies (A). Since solutions to (A) vary continuously with initial conditions, it follows that there is some $\hat{t} \in\left(t_{i}, 1\right)$ such that $\left\langle t_{i-1}, \hat{t}, 1\right\rangle$ satisfies $(\mathrm{A})$.

Now I argue that there is a backward solution to $(\mathrm{A}),\langle 1, \tilde{t}, 0\rangle$, such that $\tilde{t} \in(0,1) .{ }^{11}$ If $t_{i-1}=0$, then by the earlier construction, the sequence $\langle 1, \hat{t}, 0\rangle$ suffices; so assume that $t_{i-1}>0$. By the earlier logic, for any $\tau_{1}<1$, there is at most one value of $\tau_{2}<\tau_{1}$ such that the sequence $\left\langle 1, \tau_{1}, \tau_{2}\right\rangle$ satisfies $(\mathrm{A})$. The hypothesis that $u^{S}(y(0), 0) \neq u^{S}(y(0,1), 0)$ implies (by continuity) that for small enough $\tau_{1}$, there is no $\tau_{2}<\tau_{1}$ such that $\left\langle 1, \tau_{1}, \tau_{2}\right\rangle$ satisfies (A). Since solutions to (A) vary continuously with initial conditions, it follows that there is some $\tilde{t} \in(0,1)$ such that $\langle 1, \tilde{t}, 0\rangle$ satisfies $(\mathrm{A})$.

The proof is completed by noting that the sequence $\langle 0, \tilde{t}, 1\rangle$ defines an influential CS equilibrium partition, by construction.

The proof of Theorem 2 readily follows from the Lemmas.

Proof of Theorem 2. Necessity follows from Lemmas 1 and 2. For sufficiency, simply note that one can transform a CS equilibrium into an equilibrium of ASB by augmenting the play of $b(t)=0$ for all $t$, and $\alpha(m, b)=\alpha(m, 0)$ for any $(m, b)$ such that $b>0$.

\footnotetext{
${ }^{10}$ When Condition $\mathrm{M}$ does hold, it is well-known that the necessary and sufficient condition for influential CS equilibria is that $y(0)<y(0,1)$.

${ }^{11}$ Recall that a sequence $\left\langle s_{0}, s_{1}, \ldots, s_{N}\right\rangle$ is a backward solution to (A) if it solves (A) and $s_{1}<s_{0}$.
} 


\title{
5 Continuity of the Equilibrium Correspondence
}

At the end of their paper, ASB (p. 15) write:

\begin{abstract}
"if the costly signaling literally involves money ... imposing a budget constraint might be appropriate. A referee conjectures that for arbitrarily small budget constraints, the qualitative properties of the equilibrium set are close to those of the Crawford-Sobel model. This conjecture has strong intuition ... However, a general argument has proved elusive."
\end{abstract}

This is a statement about continuity of the equilibrium outcome correspondence. Lower hemi-continuity is straightforward: any CS equilibrium partition supports an equilibrium when burned money is available, where no type actually burns any positive amounts of money. So the real issue is that of upper hemi-continuity, i.e. as the budget of burned money shrinks, are all equilibria "close" to CS equilibria? This section shows that the answer is yes, as conjectured.

Let $\bar{b}>0$ denote the maximal amount of burned money available to the Sender. That is, the Sender's strategy is henceforth $\sigma:[0,1] \rightarrow M \times[0, \bar{b}]$. ASB (Lemma 1 and subsequent discussion) have proven that every equilibrium with burned money is partitional; the only difference with CS being that all types within an element of the partition may be completely separating rather than pooling with each other. In particular, higher Sender types elicit weakly higher actions from the Receiver.

The key step in analyzing equilibria as $\bar{b} \rightarrow 0$ is the following result which severely restricts the set of separating types for small $\bar{b}$.

Lemma 3. For any $\varepsilon>0$, there exists $\delta>0$ such that for all $\bar{b}<\delta$, the only separating types lie in $[0, \varepsilon]$.

The proof consists of minor modifications of Lemma 2 in Kartik [4]; it is included in the Appendix for completeness. 
The Lemma says that as $\bar{b}$ gets small, the measure of separating types in any equilibrium is converging to 0 , and moreover, all separation occurs in a neighborhood of type 0 . Accordingly, henceforth, given an equilibrium, $(\sigma, \alpha)(\bar{b})$, with supporting partition $\left\langle s_{0} \equiv 0, s_{1}, \ldots, s_{N} \equiv 1\right\rangle(\bar{b})$, let $\underline{s}(\bar{b}) \geq 0$ be the lowest type such that there are no separating types of positive measure above $\underline{s}(\bar{b}) .{ }^{12} \quad$ Clearly, $\underline{s}(\bar{b}) \rightarrow 0$ as $\bar{b} \rightarrow 0$. With some abuse of terminology, I will refer to the supporting partition of an equilibrium as $\left\langle s_{0} \equiv \underline{s}, s_{1}, \ldots, s_{N} \equiv 1\right\rangle(\bar{b})$.

Theorem 3. For any $\varepsilon>0$, there exists $\delta>0$ such that when $\bar{b}<\delta$, for any equilibrium supported by $\left\langle s_{0} \equiv \underline{s}, s_{1}, \ldots, s_{N} \equiv 1\right\rangle(\bar{b})$, there is a CS equilibrium supported by $\left\langle t_{0} \equiv 0, t_{1}, \ldots, t_{N} \equiv 1\right\rangle$ such that $\left|s_{j}-t_{j}\right|<\varepsilon$ for all $j \in\{0,1, \ldots, N\}$.

The proof follows from minor modifications of Lemma 2 in Kartik [4]; it is included in the Appendix for completeness.

\section{Appendix}

Proof of Theorem 1. Construct the equilibrium as follows. Pick a set of $N$ distinct messages, $\left\{m_{1}, \ldots, m_{N}\right\}$. For all $t \in\left[0, t_{N-1}\right)$ define $\sigma(t)$ as follows: $t \in\left[t_{i-1}, t_{i}\right)(i \in$ $\{1, \ldots, N-1\})$ plays $\sigma(t)=\left(m_{i}, 0\right)$. For type $t_{N-1}$, set $m\left(t_{N-1}\right)=m_{N}$ and $b\left(t_{N-1}\right)=$ $C\left(t_{N-1}\right)$ where

$$
C\left(t_{N-1}\right) \equiv \begin{cases}u^{S}\left(y\left(t_{N-1}\right), t_{N-1}\right)-u^{S}\left(y\left(t_{N-2}, t_{N-1}\right), t_{N-1}\right) & \text { if } N>1 \\ 0 & \text { if } N=1\end{cases}
$$

That is, if $N>1, b\left(t_{N-1}\right)$ is the amount of burned money that would make $t_{N-1}$ indifferent between eliciting action $y\left(t_{N-1}\right)$ (i.e. revealing itself) by burning $b\left(t_{N-1}\right)$ and eliciting $y\left(t_{N-2}, t_{N-1}\right)$ with no burned money. ${ }^{13}$ If $N=1$, then there are no types below $t_{N-1} \equiv 0$, hence $b\left(t_{N-1}\right)$ is set to 0 .

\footnotetext{
${ }^{12}$ There are two details to note. First, supporting partitions are always defined so that adjacent to any segment of separation are segments of pooling; i.e. each segment of full separation is "maximal". Second, unlike in CS, the partition supporting an equilibrium with $\bar{b}>0$ may have (countably) infinite elements. However, above $\underline{s}$, there are are only a finite number of elements.

${ }^{13}$ This follows the approach of ASB.
} 
For all types $t \in\left(t_{N-1}, 1\right]$, set $m(t)=m_{N}$ and $b(t)$ following ASB to keep each type just indifferent between revealing itself and mimicking a marginally higher type, i.e.

$$
b(t)=\int_{t_{N-1}}^{t} u_{1}^{S}(y(s), s) y^{\prime}(s) d s+C\left(t_{N-1}\right)
$$

The Receiver's response for any signal on the equilibrium path is given by $\alpha\left(m_{i}, 0\right)=$ $y\left(t_{i-1}, t_{i}\right)$ for all $i \in\{1, \ldots, N-1\}$ and $\alpha\left(m_{N}, \hat{b}\right)=y\left(b^{-1}(\hat{b})\right)$ for all $\hat{b} \in\left[b\left(t_{N-1}\right), b(1)\right]$. For signals off the equilibrium path, proceed thus: define $a_{0} \equiv \alpha(\sigma(0)) ;{ }^{14}$ for all signals $(m, b)$ such that there is no $t$ with $(m, b)=\sigma(t)$, set $\alpha(m, b)=a_{0}$.

It is straightforward to verify that these strategies constitutes an equilibrium where $b(\cdot)$ is strictly increasing on $\left[t_{N-1}, 1\right]$.

Proof of Lemma 3. Pick any type $\hat{t}>0$ and suppose it is separating. I argue to a contradiction for $\bar{b}$ small enough. Denote by $\tilde{t}$ the type such that $y^{S}(\tilde{t})=y(\hat{t})$ if it exists, or else let $\tilde{t}=0$. Note that $\tilde{t}$ is strictly smaller than $\hat{t}$ ( since $y^{S}(t)>y^{R}(t)$ for all $t$ ) and does not vary with $\bar{b}$. Since $\hat{t}$ is separating by hypothesis, $\alpha(\sigma(\tilde{t})) \leq y(\tilde{t}, \hat{t})$. For type $\tilde{t}$ not to imitate (i.e. pool with) $\hat{t}$ requires $u^{S}(y(\hat{t}), \tilde{t})-u^{S}(y(\tilde{t}, \hat{t}), \tilde{t}) \leq b(\hat{t})-b(\tilde{t})$. However, the Right Hand Side is bounded above by $\bar{b}$ whereas the Left Hand Side is a strictly positive constant; hence the inequality fails for all $\bar{b}$ smaller than some strictly positive positive threshold.

Proof of Theorem 3. By Lemma 3, for any $\varepsilon>0$, there is a $\delta>0$ such that for all $\bar{b}<\delta$, in any equilibrium partition $\left\langle s_{0} \equiv \underline{s}, s_{1}, \ldots, s_{N} \equiv 1\right\rangle(\bar{b}), s_{0}<\varepsilon$ and there are only pools above $s_{0}$. For any pooling interval $\left(s_{j-1}, s_{j}\right)$, denote the amount of burned money by all types in this pool as $b_{j}$. The incentive compatibility conditions for equilibrium require that for all $j \in\{1, \ldots, N-1\}$,

$$
u^{S}\left(y\left(s_{j-1}, s_{j}\right), s_{j}\right)-u^{S}\left(y\left(s_{j}, s_{j+1}\right), s_{j}\right)=b_{j}-b_{j+1}
$$

As $\bar{b} \rightarrow 0$, the RHS of Eq. (IC) converges to 0. It follows from equations (IC) and (A) that if for all $\bar{b}$ sufficiently small, every equilibrium partition has $s_{1}(\bar{b})$ arbitrarily close to some CS partition first segment boundary $t_{1}$, the Theorem is true.

\footnotetext{
${ }^{14}$ Since $\sigma(0)$ is an on-the-equilibrium path signal, $\alpha(\sigma(0))$ has already been defined.
} 
So suppose towards contradiction that this is not the case. Then there exists a sequence $\left\{\bar{b}_{i}\right\}_{i=1}^{\infty} \rightarrow 0$ and an equilibrium partition for each $\bar{b}_{i}$ such that $s_{1}\left(\bar{b}_{i}\right)$ converges (in subsequence) to some $\bar{s}$ that is not a CS partition first segment boundary. Consider a forward solution to the difference equation (A) starting with $\tau_{0}=0$ and $\tau_{1}=\bar{s}$. Since $\bar{s}$ is not the first segment boundary of a CS partition, there is a $\theta>0$ such that no $\tau_{j}(j=0,1, \ldots)$ lies in $(1-\theta, 1]$. Noting that for sufficiently small $\bar{b}_{i}, s_{0}\left(\bar{b}_{i}\right) \equiv \underline{s}\left(\bar{b}_{i}\right)$ and $s_{1}\left(\bar{b}_{i}\right)$ are arbitrarily close to $\tau_{0} \equiv 0$ and $\tau_{1} \equiv \bar{s}$ respectively, it follows from Eq. (IC) that each $s_{j}\left(\bar{b}_{i}\right)$ is arbitrarily close to some $\tau_{j}(j=0,1, \ldots)$. Thus, for small enough $\bar{b}_{i}$, there is no $j$ such that $s_{j}\left(\bar{b}_{i}\right)=1$. But this is a contradiction with the requirement for an equilibrium partition.

\section{References}

[1] Austen-Smith, D., And J. S. Banks (1998): "Cheap Talk and Burned Money," Center for Mathematical Studies in Economics and Management Science DP 1245, Northwestern University.

[2] (2000): "Cheap Talk and Burned Money," Journal of Economic Theory, 91(1), $1-16$.

[3] Crawford, V., and J. Sobel (1982): "Strategic Information Transmission," Econometrica, 50(6), 1431-1451.

[4] Kartik, N. (2005a): "Information Transmission with Almost-Cheap Talk," mimeo, University of California, San Diego.

[5] (2005b): "On Cheap Talk and Burned Money," mimeo, University of California, San Diego. 